\title{
Finitely Valued Indistinguishability Operators
}

\author{
Gaspar Mayor ${ }^{1}$ and Jordi Recasens ${ }^{2}$
}

1 Department of Mathematics and Computer Science, Universitat de les Illes Balears, 07122 Palma de Mallorca, Illes Balears, Spain

gmayor@uib.es

2 Secció Matemàtiques i Informàtica, ETS Arquitectura del Vallès, Universitat Politècnica de Catalunya, Pere Serra 1-15, 08190 Sant Cugat del Vallès, Spain

j.recasens@upc.edu

\begin{abstract}
Fuzzy equality relations or indistinguishability operators generalize the concepts of crisp equality and equivalence relations in fuzzy systems where inaccuracy and uncertainty is dealt with. They generate fuzzy granularity and are an essential tool in Computing with Words (CWW). Traditionally, the degree of similarity between two objects is a number between 0 and 1, but in many occasions this assignment cannot be done in such a precise way and the use of indistinguishability operators valued on a finite set of linguistic labels such as small, very much,... would be advisable. Recent advances in the study of finitely valued tnorms allow us to combine this kind of linguistic labels and makes the development of a theory of finitely valued indistinguishability operators and their application to real problems possible.
\end{abstract}

Keywords: Representation Theorem, Similarity, $T$-indistinguishability Operator, finitely valued t-norm.

\section{Introduction}

\section{$1.1 \quad$ Finitely-Valued t-Norms}

In Fuzzy Logic, the logical conjunction is modeled by a t-norm. In this way infinitely valued logics are obtained in which the truth degree of a proposition is a number between 0 and 1 . In fuzzy systems, t-norms are also used to model the intersection of fuzzy subsets that are also valued in the unit interval.

In many cases, assigning an exact and precise value between 0 and 1 is not realistic since due to linguistic vagueness or lack of precision in the data this assignment is necessarily imprecise. It would be more reasonable in these cases to consider only a totally ordered finite chain (that can be identified with a finite subset of $[0,1])$ to valuate the fuzzy concepts.

So the study of operators defined on a finite chain $L$ is of great interest, especially because reasoning is usually done by using linguistic terms or labels that are totally ordered. For instance, the size of an object can be granularized in very small, small, average, big, very big. If there is defined an operator $T$ on this set, then we will be able to combine these labels in order to obtain the 
combined one, e.g. T(average, very big). In this way of tackling the problem of combining labels, the calculations are very simple since there is no need neither to assign numerical values to them, nor to identify them with an interval or with a fuzzy subset.

Another case when there is useful to use finite chains is when the valued are discrete by nature or by discretization. On a survey of satisfaction of the clients with respect to some service, they may be asked to evaluate it with a natural number between 0 and 5 or with labels going from not at all satisfied to very satisfied.

In this line, different authors have translated t-norms and t-conorms to finite chains $([5,6])$ obtaining interesting theoretical results.

\subsection{Finitely Valued Indistinguishability Operators}

In almost all situations the human being categorizes or granularizes the properties or features of the objects in a finite set $L$ of linguistic labels that can be linearly ordered. In these cases, these properties are evaluated on $L$ in a natural way and consequently the fuzzy subsets of the universe of discourse are also valued on $L$.

In the same way, the degree of similarity, equivalence or indistinguishability between two objects is not a numerical value between 0 and 1 , but an element of $L$ that can be interpreted as rather, very much,

Indistinguishability operators valued in these finite chains seem to be a very interesting tool that will allow us to study the similarity between objects taking into account the granularity generated by $L$ and will give an interpretation to the calculation on this chain.

The degree of similarity or indistinguishability $E(x, y)$ between two objects $x$ and $y$ will be bounded by the corresponding degrees to $E(x, z)$ and $E(y, z)$.

\subsection{Organization of the Paper}

After this introductory section a section of preliminary concepts of finitely valued t-norms follows. Section 3 is devoted to some properties of finitely valued indistinguishability operators. In particular, the Representation Theorem [9] is generalized to these operators. Section 4 is devoted to additive generators of finitely valued t-norms. Most of them have additive generators and a new pseudo inverse is defined in order to be able to generate their residuation. The results are applied in section 5 to find the dimension and a basis of finitely valued indistinguishability operators. A section of Concluding Remarks ends the work.

\section{Preliminaries}

This section contains some definitions and results on finitely valued t-norms that will be needed later on the paper.

Let $L$ be a finite totally ordered set with minimum $e$ and maximum $u$. 
Definition 1. A binary operation $T: L \times L \rightarrow L$ is a $t$-norm if and only if for all $x, y, z \in L$

1. $T(x, y)=T(y, x)$

2. $T(T(x, y), z)=T(x, T(y, z))$

3. $T(x, y) \leq T(x, z)$ whenever $y \leq z$

4. $T(x, u)=x$

The set of t-norms on a finite chain depends only on its cardinality. For this reason we will only consider the chains $L=\{0,1, \ldots, n\}$ and $L^{\prime}=\left\{0=\frac{0}{n}, \frac{1}{n}, \frac{2}{n}, \ldots\right.$, $\left.\frac{n}{n}=1\right\}$.

\section{Example 1}

1. The Minimum t-norm $T_{M}$ on $L$ is defined by $\left.T_{M}(i, j)=\min \{i, j\}\right)$.

2. The Eukasiewicz t-norm $T_{\mathrm{Ł}}$ on $L$ is defined by $T_{\mathrm{Ł}}(i, j)=\max (i+j-n, 0)$.

Smooth t-norms on finite chains are the equivalent of continuous ones defined on $[0,1]$.

Definition 2. A map $f: L \rightarrow L$ is smooth if and only if

$$
0 \leq f(i+1)-f(i) \leq 1 \text { for all } i \in L, i<n \text {. }
$$

Definition 3. A map $F: L \times L \rightarrow L$ is smooth if and only if it is smooth with respect to both variables.

Definition 4. A t-norm $T$ on $L$ is divisible if and only if for all $i, j \in L$ with $i \leq j$ there exists $k \in L$ such that

$$
i=T(j, k)
$$

Smoothness and divisibility are equivalent concepts for t-norms.

Proposition 1. A t-norm on $L$ is smooth if and only if it is divisible.

The next proposition characterizes all smooth t-norms on $L$ as particular ordinal sums of copies of the t-norm of Eukasiewicz.

Proposition 2. A t-norm $T$ on $L$ is smooth if and only if there exists $J=\{0=$ $\left.i_{0}<i_{1}<\ldots<i_{m}=n\right\} \subseteq L$ such that

$$
T(i, j)= \begin{cases}\max \left(i_{k}, i+j-i_{k}\right) & \text { if } i, j \in\left[i_{k}, i_{k+1}\right] \text { for some } i_{k} \in J \\ \min \{i, j\} & \text { otherwise. }\end{cases}
$$

$T$ is said to be an ordinal sum and can be represented by $T=<0=i_{0}, i_{1}, \ldots i_{m}=$ $n>$. 


\section{$3 \quad$ Finitely Valued Indistinguishability Operators}

Indistinguishability operators fuzzify the concepts of crisp equality and crisp equivalence relation. They have been studied under different settings, mainly valued on $[0,1]$ and with respect to a left continuous t-norm, though some generalizations to more general structures like GL-monoids have been carried on.

A very important result is the Representation Theorem that roughly speaking says that every fuzzy set $\mu$ on a universe $X$ generates an indistinguishability operator $E_{\mu}$ and that every indistinguishability operator on $X$ can be obtained as the infimum of a family of indistinguishability operators generated in this way. The theorem was first proved by Ovchinnikov for the product t-norm. Then it was generalized to continuous t-norms by Valverde and in 2 it is noticed that it is also true for GL-monoids. Since finitely valued t-norms are such monoids, the Representation Theorem also applies to them.

This section adapts the basic definitions on indistinguishability operators to the finite valued case. In particular, the Representation Theorem and the idea of extensionality are recalled.

Also the concepts of dimension and basis of an indistinguishability operator are considered and the characterization of the set of extensional fuzzy subsets with respect to an indistinguishability operator is adapted to the context of finitely valued t-norms.

Definition 5. Let $T$ be a t-norm on L. Its residuation $\vec{T}$ is defined by

$$
\vec{T}(i \mid j)=\max \{k \in L \mid T(i, k) \leq j\} .
$$

\section{Example 2}

1. If $T_{\mathrm{E}}$ is the Eukasiewicz t-norm on $L$, then $\overrightarrow{T_{\mathrm{E}}}(i \mid j)=\max (0, n-i+j)$ for all $i, j \in L$.

2. If $T_{M}$ is the Minimum t-norm on $L$, then $\overrightarrow{T_{M}}(i \mid j)=$

$$
\begin{cases}\min \{i, j\} & \text { if } i>j \\ n & \text { otherwise. }\end{cases}
$$

Definition 6. The biresiduation $E_{T}$ associated to a given $t$-norm $T$ on $L$ is defined by

$$
E_{T}(i, j)=T(\vec{T}(i \mid j), \vec{T}(j \mid i)) .
$$

Example 3

1. If $T_{\mathrm{E}}$ is the Eukasiewicz t-norm on $L$, then $E_{T_{\mathrm{E}}}(i, j)=n-|i-j|$ for all $i, j \in L$.

2. If $T_{M}$ is the Minimum t-norm, then $E_{T_{M}}(i, j)=$

$$
\begin{cases}\min \{i, j\} & \text { if } i \neq j \\ n & \text { otherwise. }\end{cases}
$$


Proposition 3. Let $T=<0=i_{0}, i_{1}, \ldots i_{m}=n>$ be a smooth $t$-norm on L. Its residuation $\vec{T}$ is

$$
\vec{T}(i \mid j)= \begin{cases}n & \text { if } i \leq j \\ \max \left(0, i_{k+1}-i+j\right) & \text { if } i, j \in\left[i_{k}, i_{k+1}\right] \text { for some } i_{k} \in J \text { and } i>j \\ j & \text { otherwise. }\end{cases}
$$

Proposition 4. Let $T=<0=i_{0}, i_{1}, \ldots i_{m}=n>$ be a smooth $t$-norm on L. Its biresiduation $E_{T}$ is

$$
E_{T}(i, j)= \begin{cases}n & \text { if } i=j \\ i_{k+1}-|i+j| & \text { if } i, j \in\left[i_{k}, i_{k+1}\right] \text { for some } i_{k} \in J \\ \min \{i, j\} & \text { otherwise. }\end{cases}
$$

$E_{T}$ is a special kind of $T$-indistinguishability operator.

Definition 7. Given a t-norm $T$ on $L$, a $T$-indistinguishability operator $E$ on a set $X$ is a fuzzy relation $E: X \times X \rightarrow L$ satisfying for all $x, y, z \in X$

1. $E(x, x)=n$ (Reflexivity)

2. $E(x, y)=E(y, x)$ (Symmetry)

3. $T(E(x, y), E(y, z)) \leq E(x, z)$ (T-transitivity).

Proposition 5. The biresiduation $E_{T}$ of a $t$-norm $T$ on $L$ is a $T$-indistinguishability operator on $L$.

Theorem 1. Representation Theorem for T-indistinguishability operators. Let $R$ be a fuzzy relation on a set $X$ and $T$ a t-norm on $L . R$ is a T-indistinguishability operator if and only if there exists a family $\left(\mu_{i}\right)_{i \in I}$ of L-fuzzy subsets of $X$ (i.e.: $\mu_{i}: X \rightarrow L$ for all $x \in X$ ) such that for all $x, y \in X$

$$
R(x, y)=\inf _{i \in I} E_{T}\left(\mu_{i}(x), \mu_{i}(y)\right) .
$$

$\left(\mu_{i}\right)_{i \in I}$ is called a generating family of $R$ and a fuzzy subset that belong to a generating family of $R$ is called a generator of $R$.

Extensional fuzzy subsets with respect to a $T$-indistinguishability operator $E$ play a central role since they are the only observable sets taking $E$ into account. In the crisp case, when $E$ is a crisp equivalence relation on a universe $X$, the only crisp subsets from which something can be said if $E$ is considered are only the union of equivalence classes of $E$ (and intersections if we want to add the empty set). The equivalence classes give the granularity in $X$. If $E$ is a fuzzy relation, extensional sets play this role and they show the granularity generated by $E$.

Definition 8. Let $T$ be a t-norm on $L, E$ be a $T$-indistinguishability operator on a set $X$ and $\mu$ a fuzzy subset of $X . \mu$ is extensional with respect to $E$ if and only if for all $x, y \in X$

$$
T(E(x, y), \mu(x)) \leq \mu(y) .
$$

$H_{E}$ will denote the set of all extensional fuzzy subsets with respect to $E$. 
It can be proved that a fuzzy subset is extensional with respect to a $T$ indistinguishability operator $E$ if and only if it is a generator of $E$.

The next result is then straightforward.

Proposition 6. Let $T$ be a t-norm on $L, E$ be a $T$-indistinguishability operator on a set $X$ and $\mu$ a fuzzy subset of $X . \mu$ is extensional with respect to $E$ if and only if $E_{\mu} \geq E$.

In 2 there is a nice characterization of $H_{E}$.

Proposition 7. Let $F(X)$ be the set of all fuzzy subsets of $X$ and $T$ a t-norm on L. Given a set $H$ of fuzzy subsets of $X$, there exists a $T$-indistinguishability operator $E$ on $X$ such that $H=H_{E}$ if and only if for all fuzzy subsets $\mu$ of $H$ and for all $\alpha \in[0,1]$,

1. $T(\alpha, \mu) \in H$

2. $\vec{T}(\alpha \mid \mu) \in H$

3. $\vec{T}(\mu \mid \alpha) \in H$

4. $(H, \leq)$ is a complete sub lattice of $(F(X), \leq)$.

Going back th the Representation theorem 1, different families of fuzzy subsets can generate the same $T$-indistinguishability operator $E$. This gives great interest to the theorem, since if we interpret the elements of the family as degrees of matching between the elements of $X$ and a set of prototypes, we can use different features, giving different interpretations to $E$.

Among the generating families of a relation, the ones with low cardinality are of special interest, since they have an easy semantical interpretation and also because the information contained in its matrix can be packed in a few fuzzy subsets.

Definition 9. Let $T$ be a t-norm on $L$ and $E$ a $T$-indistinguishability operator on $X$. The dimension of $E$ is the minimum of the cardinalities of the generating families of $E$ in the sense of the Representation Theorem. A generating family with this cardinality is called a basis of $E$.

A geometric approach and an algorithm for calculating the dimension and a basis of $T$-indistinguishability operators with $T$ continuous Archimedean or the Minimum t-norm in $[0,1]$ can be found in [1].

In Section [5 an algorithm to find dimensions and basis of $T$-indistinguishability operators for an additively generated t-norm $T$ on $L$ will be provided.

\section{Additive Generators}

Contrarily to the case of t-norms defined on $[0,1]$, many of the t-norms on a finite chain $L$ can be additively generated. In particular, it can be proved that all smooth t-norms on $L$ - including the minimum t-norm and all ordinal sums - have an additive generator. This will provide us of a technique to find the dimension and a basis of a finitely valued $T$-indistinguishability operator $E$ as well as its set $H_{E}$ of generators or extensional sets. 
Definition 10. Let $f: L \rightarrow[0, \infty)$ be a strictly decreasing function with $f(n)=0$.

- The pseudo inverse $f_{+}^{(-1)}:[0, \infty) \rightarrow L$ is defined by

$$
f_{+}^{(-1)}(t)=\min \{i \in L ; f(i) \leq t\}=\min f^{-1}([0, t])
$$

- The pseudo inverse $f_{-}^{(-1)}:(-\infty, \infty) \rightarrow L$ is defined by

$$
f_{-}^{(-1)}(t)= \begin{cases}\max \{i \in L ; f(i) \geq t\}=\max f^{-1}([t, n]) & \text { if } t \geq 0 \\ n & \text { otherwise. }\end{cases}
$$

The first pseudo inverse $f_{+}^{(-1)}$ was first defined in $[6] \cdot f_{-}^{(-1)}$ is a new pseudo inverse introduced in this paper in order to generate the residuation and biresiduation of a t-norm on $L$.

Definition 11. Let $T$ be a t-norm on $L . T$ is generated by a strictly decreasing function $f: L \rightarrow[0, \infty)$ with $f(n)=0$ if and only if

$$
T(i, j)=f_{+}^{(-1)}(f(i)+f(j)) \text { for all } i, j \in L .
$$

$f$ is called an additive generator of $T$ and we will write $T=\langle f\rangle$.

For an additive generator $f$, we will indicate $f=\left(a_{0}, a_{1}, a_{2}, \ldots, a_{n}=0\right)$ where $a_{i}=f(i), i \in L$.

\section{Example 4}

- An additive generator of the t-norm of Eukasiewicz on $L$ is $(n, n-1, n-$ $2, \ldots, 1,0)$.

- An additive generator of the minimum t-norm $L$ is $\left(2^{n}-1,2^{n-1}-1,2^{n-2}-\right.$ $2, \ldots, 7,3,1,0)$.

Some results on additive generators are the following ones.

Proposition 8. Let $f=\left(a_{0}, a_{1}, a_{2}, \ldots, a_{n}=0\right)$ and $g=\left(b_{0}, b_{1}, b_{2}, \ldots, b_{n}=0\right)$ be strictly decreasing functions on $L$. Then $\langle f\rangle=\langle g\rangle$ if and only if for all $i, j, k \in L$ with $k \neq 0$,

1. $a_{i}+a_{j} \geq a_{0} \Rightarrow b_{i}+b_{j} \geq 0$

2. $a_{k} \leq a_{i}+a_{j}<a_{k-1} \Rightarrow b_{k} \leq b_{i}+b_{j}<b_{k-1}$.

Corollary 1. If $f: L \rightarrow[0, \infty)$ is a strictly decreasing function with $f(n)=0$ and $\lambda>0$, then $\langle f\rangle=\langle\lambda f\rangle$.

Of course, the reciprocal of the corollary is not true.

Proposition 9. If $T$ is a t-norm on $L$ with additive generator, then we can find an additive generator $f$ of $T$ with Ran $f \in Z^{+}$. 
Proposition 10. All smooth $t$-norms on $L$ have an additive generator.

For additively generated t-norms we have representations for their residuations and biresiduations.

Proposition 11. Let $T$ be a $t$-norm on $L$ with additive generator $f$. Then

$$
\vec{T}(i \mid j)=f_{-}^{(-1)}(f(j)-f(i)) \text { for all } i, j \in L .
$$

Proof. Given $i, j \in L$,

$$
\begin{aligned}
\vec{T}(i \mid j) & =\max \{k \in L \mid T(i, k) \leq j\} \\
& =\max \left\{k \in L \mid f_{+}^{(-1)}(f(i)+f(k)) \leq j\right\} \\
& =f_{-}^{(-1)}(f(j)-f(i)) .
\end{aligned}
$$

Proposition 12. Let $T$ be a $t$-norm on $L$ with additive generator $f$. Then

$$
E_{T}(i, j)=f_{-}^{(-1)}(|f(i)-f(j)|) \text { for all } i, j \in L .
$$

Proof

$$
\begin{aligned}
E_{T}(i, j) & =\min \{\vec{T}(i \mid j), \vec{T}(j \mid i)) \\
& =\min \left(f_{-}^{(-1)}(f(j)-f(i)), f_{-}^{(-1)}(f(i)-f(j))\right\} \\
& =f_{-}^{(-1)}(|f(i)-f(j)|) .
\end{aligned}
$$

\section{Dimension and Basis of an Indistinguishability Operator}

In this section we will give a method to calculate the dimension an a basis of a $T$-indistinguishability operator $E$ on a finite set $X$ when $T$, a t-norm on $L$, can be additively generated.

Let $\mu$ be a fuzzy subset of a finite set $X=\left\{r_{1}, r_{2}, \ldots, r_{s}\right\}$ of cardinality $s$. We will write $\mu=\left(q_{1}, q_{2}, \ldots, q_{s}\right)$ when $\mu\left(r_{i}\right)=q_{i}, i=1,2, \ldots, s$.

A fuzzy subset of $X$ is a generator of $E$ if and only if $E_{\mu}\left(r_{i}, r_{j}\right) \geq E\left(r_{i}, r_{j}\right)$ for all $i, j=1,2, \ldots, s$. If $T$ has $f$ as an additive generator, then this condition can be written as

$$
f_{-}^{(-1)}\left(\left|f\left(\mu\left(r_{i}\right)\right)-f\left(\mu\left(r_{j}\right)\right)\right|\right) \geq E\left(r_{i}, r_{j}\right) \text { for all } i, j=1,2, \ldots, s
$$

or

$$
\left|f\left(\mu\left(r_{i}\right)\right)-f\left(\mu\left(r_{j}\right)\right)\right| \leq f\left(E\left(r_{i}, r_{j}\right)\right) \text { for all } i, j=1,2, \ldots, s .
$$

This is equivalent to

$$
f\left(\mu\left(r_{i}\right)\right)-f\left(\mu\left(r_{j}\right)\right) \leq f\left(E\left(r_{i}, r_{j}\right)\right) \text { for all } i, j=1,2, \ldots, s .
$$


Proposition 13. Let $T$ be a t-norm on $L$ with additive generator $f$ and $E$ a $T$-indistinguishability operator on a finite set $X$ of cardinality $s$. A fuzzy subset $\mu=\left(x_{1}, x_{2}, \ldots, x_{s}\right)$ is a generator of $E$ if and only if

$$
f\left(x_{i}\right)-f\left(x_{j}\right) \leq f\left(E\left(r_{i}, r_{j}\right)\right) \text { for all } i, j=1,2, \ldots, s .
$$

In other words, $H_{E}$ is the subset of $L^{s}$ of solutions of the last system of Diophantine inequalities.

Example 5. If $T$ is the Eukasiewicz t-norm on $L$, then the last system of inequalities becomes

$$
x_{i}-x_{j} \leq n-E\left(r_{i}, r_{j}\right) \text { for all } i, j=1,2, \ldots, s .
$$

Example 6. If $T$ is the minimum t-norm on $L$, then the last system of inequalities becomes

$$
2^{n-x_{i}}-2^{n-x_{j}} \leq 2^{n-E\left(r_{i}, r_{j}\right)}-1 \text { for all } i, j=1,2, \ldots, s .
$$

Example 7. The following fuzzy relation $E$ on $X=\left\{r_{1}, r_{2}, r_{3}, r_{4}\right\}$ is a $T_{M^{-}}$ indistinguishability operator with $L=\{0,1,2\}$.

$$
E=\left(\begin{array}{llll}
2 & 1 & 0 & 0 \\
1 & 2 & 0 & 0 \\
0 & 0 & 2 & 1 \\
0 & 0 & 1 & 2
\end{array}\right) .
$$

An $L$-fuzzy subset $\left(x_{1}, x_{2}, x_{3}, x_{4}\right)$ of $X$ is a generator of $E$ if and only if it satisfies the following Diophantine system of inequations.

$$
\begin{aligned}
& 2^{2-x_{1}}-2^{2-x_{2}} \leq 2^{2-1}-1 \\
& 2^{2-x_{1}}-2^{2-x_{3}} \leq 2^{2}-1 \\
& 2^{2-x_{1}}-2^{2-x_{4}} \leq 3 \\
& 2^{2-x_{2}}-2^{2-x_{1}} \leq 1 \\
& 2^{2-x_{2}}-2^{2-x_{3}} \leq 3 \\
& 2^{2-x_{2}}-2^{2-x_{4}} \leq 3 \\
& 2^{2-x_{3}}-2^{2-x_{1}} \leq 3 \\
& 2^{2-x_{3}}-2^{2-x_{2}} \leq 3 \\
& 2^{2-x_{3}}-2^{2-x_{4}} \leq 1 \\
& 2^{2-x_{4}}-2^{2-x_{1}} \leq 3 \\
& 2^{2-x_{4}}-2^{2-x_{2}} \leq 3 \\
& 2^{2-x_{4}}-2^{2-x_{3}} \leq 1
\end{aligned}
$$

$H_{E}$ has 26 elements:

$$
\begin{gathered}
H_{E}=\{(2,2,2,2),(2,2,2,1),(2,2,1,2),(2,2,1,1),(2,2,0,0),(2,1,2,2),(2,1,2,1), \\
(2,1,1,2),(2,1,1,1),(2,2,0,0),(2,1,0,0),(1,2,2,2),(1,2,2,1),(1,2,1,2), \\
(1,2,1,1),(1,2,0,0),(1,1,2,2),(1,1,2,1),(1,1,1,2),(1,1,1,1),(1,1,0,0), \\
(0,0,1,2),(0,0,2,1),(0,0,2,2),(0,0,1,1),(0,0,0,0)\}
\end{gathered}
$$

$E$ has dimension 2 and $\{(1,2,0,0),(0,0,1,2)\}$ is a basis of $E$. 


\section{Concluding Remarks}

In this work finitely valued indistinguishability operators have been introduced. The most relevant results are

- A new pseudo inverse has been defined that allow us to generate the residuation of a t-norm.

- A method to find the dimension and a basis of a $T$-indistinguishability operator solving a Diophantine system of inequalities has been developed.

These results will be related to infinitely valued indistinguishability operators in forthcoming works by the authors.

Acknowledgments. Research partially supported by project numbers TIN200614311, TIN2009-07235, MTM2009-10962 and by grant PR2009-0079.

\section{References}

1. Boixader, D., Jacas, J., Recasens, J.: Fuzzy Equivalence Relations: Advanced Material. In: Dubois, Prade (eds.) Fundamentals of Fuzzy Sets, pp. 261-290. Kluwer, Dordrecht (2000)

2. Castro, J.L., Klawonn, F.: Similarity in Fuzzy Reasoning. Mathware \& Soft Computing (1996)

3. Jacas, J.: Similarity relations - the calculation of minimal generating families. Fuzzy Sets and Systems 35, 151-162 (1990)

4. Ling, C.M.: Representation of associative functions. Publ. Math. Debrecen 12, 189 $212(1965)$

5. Mas, M., Monserrat, M., Torrens, J.: QL-Implications on a finite chain. In: Proc. Eusflat 2003, Zittau, pp. 281-284 (2003)

6. Mayor, G., Torrens, J.: Triangular norms on discrete settings. In: Klement, E.P., Mesiar, R. (eds.) Logical, Algebraic, Analytic, and Probabilistic Aspects of Triangular Norms, pp. 189-230. Elsevier BV, Amsterdam

7. Klement, E.P., Mesiar, R., Pap, E.: Triangular norms. Kluwer, Dordrecht (2000)

8. Schweizer, B., Sklar, A.: Probabilistic Metric Spaces. North-Holland, Amsterdam (1983)

9. Valverde, L.: On the structure of F-indistinguishability operators. Fuzzy Sets and Systems 17, 313-328 (1985)

10. Zadeh, L.A.: Similarity relations and fuzzy orderings. Information Science 3, 177200 (1971)

11. Zadeh, L.A.: Fuzzy logic=Computing with words. IEEE Transactions on Fuzzy Systems 90, 103-111 (1996)

12. Zadeh, L.A.: Towards a theory of fuzzy information granulation and its centrality in human reasoning and fuzzy logic. Fuzzy Sets and Systems 90, 111-127 (1997) 